\title{
Right Salpingo-Oophorectomy
}

National Cancer Institute

\section{Source}

National Cancer Institute. Right Salpingo-Oophorectomy. NCI Thesaurus. Code C51926.

Surgical removal of the right fallopian tube and right ovary. 